\title{
SHOCK CHEMISTRY IN BIPOLAR MOLECULAR OUTFLOWS
}

\author{
R. BACHILLER \& M. PÉREZ GUTIÉRREZ \\ IGN Observatorio Astronómico Nacional \\ Apartado 1143, E-28800 Alcalá de Henares, Spain
}

\begin{abstract}
Chemical studies have a great potential to study the structure and evolution of the bipolar molecular outflows driven by young stellar objects. In this paper, we discuss some very recent mm-wave studies of $\mathrm{L} 1157$, a bipolar molecular outflow driven by a Class 0 protostar. These observations are very useful to illustrate the chemical alterations produced by a violent highly-collimated outflow. Different molecular lines are observed to trace different components of the gas. Some molecules are abundant in the quiescent medium but are not observed in the shock (e.g. $\mathrm{C}_{3} \mathrm{H}_{2}, \mathrm{~N}_{2} \mathrm{H}^{+}$, $\mathrm{H}^{13} \mathrm{CO}^{+}, \mathrm{DCO}^{+}$), whereas some otherwise rare molecules are very enhanced at the shocked region (e.g. $\mathrm{SiO}, \mathrm{CH}_{3} \mathrm{OH}, \mathrm{H}_{2} \mathrm{CO}, \mathrm{HCN}, \mathrm{CN}, \mathrm{SO}, \mathrm{SO}_{2}$ ). In addition, we have observed strong gradients in the chemical composition across the outflow blue lobe. We briefly discuss the chemistry of the most important molecules, devoting special attention to the species which are thought to be abundant in interstellar ice mantles.
\end{abstract}

\section{Introduction: chemistry and star-forming regions}

One of the main contributions of radioastronomy to astrophysics is the discovery that there is a very rich chemistry operating in the interstellar medium (ISM). Up to 120 molecular species are presently known in the ISM, and this number increases at a rate of 4 new molecules identified per year in average. Interstellar chemistry involves processes in gas phase and on the surfaces of dust grains, and it is very different from the chemistry on Earth (including laboratory chemistry). Besides the extreme conditions found in the ISM, the time scales are so long that the chemical equilibrium can be attained in many situations. However, transient phenomena should 
also be present, in particular in regions subjected to the passage of shock waves.

Radio observations of different chemical species have been used for a long time to diagnose the physical conditions in star forming regions. Different species can probe different cloud regions, and help to provide a complete description of the whole cloud. Essentially for the same reasons, chemical studies have a great potential to understand the structure and evolution of the bipolar outflows driven by young stellar objects (YSOs).

\section{Outflows from Class 0 protostars}

Bipolar molecular outflows are observed in virtually all YSOs (e.g. Bachiller 1996), even around the youngest protostars already identified (the so-called "Class 0" objects, André et al. 1993). In fact, outflows from Class 0 sources are particularly violent (Bontemps et al. 1996): the mechanical power can reach $30 \%$ of the bolometric luminosity of the central engine instead of the $0.001-0.01 \%$ observed in most standard outflows. Such violent outflows accompanying the birth of a new star impact on the ambient cloud dispersing the surrounding molecular material, and determining the evolution of the dense core where the star was born. Outflows drive shock waves that compress and heat the gas triggering important chemical processes that do not operate in quiescent environments, producing dramatic effects on the structure and composition of the surrounding molecular cloud. In addition, outflows from Class 0 sources are highly collimated (Bachiller 1996), so the region where the shock occurs is well defined spatially, and well separated from the vicinity of the driving source which keeps the properties of the quiescent ambient gas. Processes such as endothermic reactions, sublimation of ices, and disruption of dust grain cores lead to a "shock-chemistry" which is radically different from that operating in the quiescent medium. Some of these processes are fast, and the cooling times of the shocked region are short, making the chemistry strongly time-dependent. Thus, the study of different chemical species can in principle be used as an indication of the state of evolution of the bipolar outflow and of its driving YSO.

Recent observations with high angular resolution and sensitivity are providing important insights in these transient phenomena. For instance, in the case of the outflow around IRAS $03282+3035$, the $\mathrm{SiO}$ emission is only detected at the end of the outflow lobe, where there is the maximum interaction of the outflow with the ambient gas (Bachiller et al. 1994). In the case of $\mathrm{L} 1448$, the $\mathrm{SiO}$ emission is associated with the high-velocity molecular "bullets" at the main outflow axis (Bachiller et al. 1991), probably tracing shocks produced by the central jet (Dutrey et al. 1997). In both cases, no $\mathrm{SiO}$ emission is observed at ambient velocities. 
To summarize, shocks have detectable, and even spectacular, manifestations in the chemical composition of the gas. Outflows from Class 0 protostars are of the highest interest to study the chemical alterations caused by shocks. The observations of different chemical species can provide the physical conditions in different regions of the shock. Due to its time-dependence, shock-chemistry has a great potential to be used as a clock to study the evolution of outflows.

\section{L 1157-mm as an example of Class 0 source}

In the following, we will highlight one of the most illustrative cases of outflows from Class 0 sources: the bipolar molecular outflow in the dark cloud L 1157. Due to its proximity (440 pc), high collimation, favorable orientation in the sky, and high column densities in the shocked region, this is one of the best objects to study the rich phenomenology associated with young bipolar outflows, in particular the effects of the shock-chemistry.

The highly collimated molecular outflow in L 1157 was discovered by Umemoto et al. (1992). The outflow is driven by L 1157-mm (IRAS 20386 +6751 ), a very cold source of about $11 \mathrm{~L}_{\odot}$, embedded within a dense $\mathrm{NH}_{3}$ core (Bachiller et al. 1993). The central source presents all the attributes of Class 0 sources, including a relatively strong flux density at millimeter wavelengths due to thermal dust emission. Such high flux density makes it possible to obtain detailed images of the protostellar vicinity with $\mathrm{mm}$ wave interferometers. A recent high-angular resolution study by Gueth et al. (1997) shows that the continuum emission near $\lambda 2.7 \mathrm{~mm}$ consists of a $35 \mathrm{mJy}$ source of size $\leq 1^{\prime \prime}$, surrounded by spatially-extended low-level emission. The extended emission seems to arise from the heated edges of the cavity excavated by the high-velocity bipolar outflow. High-angular resolution images of the source in the $\mathrm{J}=1-0$ line of $\mathrm{CO},{ }^{13} \mathrm{CO}$, and $\mathrm{C}^{18} \mathrm{O}$ lead to a picture in which the $\mathrm{C}^{18} \mathrm{O}$ emission is associated with the protostellar condensation, whereas the ${ }^{13} \mathrm{CO}$ line emission mostly originates from a more extended envelope and the limb-brightened edges of the outflow. The $\mathrm{CO}$ line wings trace the bulk of the outflowing material. Possible gravitational infall motions are recognized by comparing the self-absorbed ${ }^{13} \mathrm{CO}$ profiles with the narrow $\mathrm{C}^{18} \mathrm{O}$ lines.

\section{The CO bipolar outflow in $\mathrm{L} \mathbf{1 1 5 7}$}

The bipolar outflow in L 1157 has a size of about $0.6 \mathrm{pc}$, and it is highly collimated but asymmetric (Umemoto et al. 1992). Figure 1 shows a CO $2 \rightarrow 1$ integrated intensity map of the whole outflow which was recently obtained at the IRAM 30-m telescope. It appears that the southern blue-shifted lobe is more compact and brighter than the northern lobe, suggesting that 


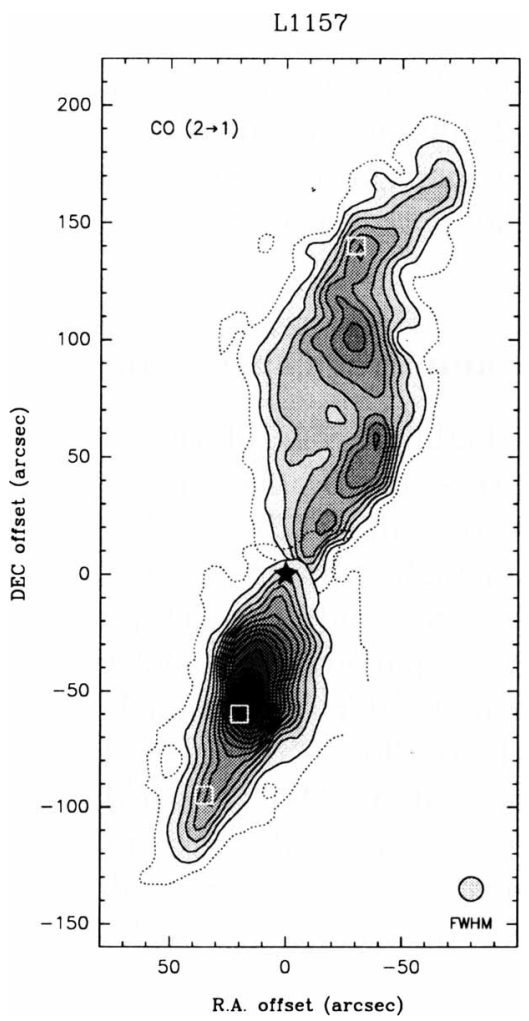

Figure 1. CO $2 \rightarrow 1$ emission from the $\mathrm{L} 1157$ outflow integrated over velocity intervals from -20 to $3 \mathrm{~km} / \mathrm{s}$ (blueshifted gas at the South of the driving source) and from 3 to 30 $\mathrm{km} / \mathrm{s}$ (redshifted gas toward the North of the source). Position offsets are in arcsec with respect L $1157-\mathrm{mm}$, the outflow exciting source. First contour and step are $11 \mathrm{~K} \mathrm{~km} / \mathrm{s}$. The squares mark the positions in which we carried out a survey of molecular lines (see text).

the blue-shifted gas is shocking against some kind of obstacle, whereas the northern lobe is flowing more freely. The brightness of the blue-shifted lobe makes it one of the best places to study the structure of the shock. Gueth et al. (1996) obtained an interferometric high-resolution CO $1 \rightarrow 0$ image of the blueshifted lobe. Such image reveals at least two prominent limbbrightened cavities which seem to be created by the propagation of large bow shocks. Interestingly, the two cavities are not well aligned on a single line passing through the exciting source, L $1157-\mathrm{mm}$, as if the axis of the underlying jet had precessed from the first ejection event to the second one. A simple spatio-kinematic model in which the jet precesses on a narrow cone (of opening angle close to $6^{\circ}$ ) provides an accurate description of the observations. 
Indeed the sharp increase of the $\mathrm{CO}$ brightness temperature towards the blueshifted lobe in L 1157 could be due to the gas heating produced by the shock. Multiline observations of ammonia, the best interstellar thermometer, confirm that this is the case, and provide a good estimate of the gas kinetic temperature in the shock, which results to be in the range 60-100 $\mathrm{K}$ (Bachiller et al. 1993). The bow shocks at the head of the cavities are particularly well observed in $\mathrm{NH}_{3}(3,3)$ emission, and VLA images (Tafalla \& Bachiller 1995, 1997) reveal the hot regions of the bow shock structures seen in the high-velocity CO. The ammonia abundance can be estimated in both the quiescent gas and in the high-velocity outflow, and it results that this abundance is enhanced by at least a factor of 10 in the shocked region.

\section{A chemical survey of the $L \mathbf{1 1 5 7}$ outflow}

In addition to ammonia, some other molecular abundances have been long suspected to be enhanced as a result of the action of bipolar outflows on the surrounding gas. One of the most extreme examples is $\mathrm{SiO}$, whose gas phase abundance is known to be enhanced by several orders of magnitude at the heads and along the axes of some molecular outflows (Bachiller et al. 1991; Martín-Pintado et al. 1992; McMullin et al. 1994). The case of L 1157 is not less spectacular, and a wealth of observations (Mikami et al. 1992, Zhang et al. 1995, Avery \& Chiao 1996) indicate that SiO is enhanced by a factor of $\sim 10^{6}$ in the shocked region.

Spectral line surveys at $\mathrm{mm}$ wavelengths are recognized to be the best tool to explore the chemical composition of the vicinity of low-mass YSOs. Recent studies have considered two important Class 0 sources: NGC1333 IRAS4 (Blake et al. 1995) and IRAS16293 (Blake et al. 1994; van Dishoeck et al. 1995). Very recently, we have carried out a survey of the blueshifted lobe in L 1157 to study the chemical composition of the gas affected by the passage of the shock (Bachiller \& Pérez Gutiérrez 1997). We observed about 50 different lines of 27 molecules (including rare isotopic species) toward the position of the central source $(0,0)$ and several positions at the shocked regions (three of which are indicated as white squares in Fig. 1), in particular the two prominent $\mathrm{NH}_{3}$ peaks of the blueshifted lobe: $\mathrm{B} 1$ at position $\left(20^{\prime \prime},-60^{\prime \prime}\right)$, and B2 at $\left(35^{\prime \prime},-95^{\prime \prime}\right)$. Some of the profiles are shown in Fig. 2. Indeed, the narrow line profiles observed toward the position of the source arise from cold quiescent gas, whereas toward the bow shock region the profiles are dominated by the broad lines associated with the shock.

One of the first results emerging from these observations is that some molecular lines such as those of $\mathrm{C}_{3} \mathrm{H}_{2}, \mathrm{~N}_{2} \mathrm{H}^{+}, \mathrm{H}^{13} \mathrm{CO}^{+}$, and $\mathrm{DCO}^{+}$are 


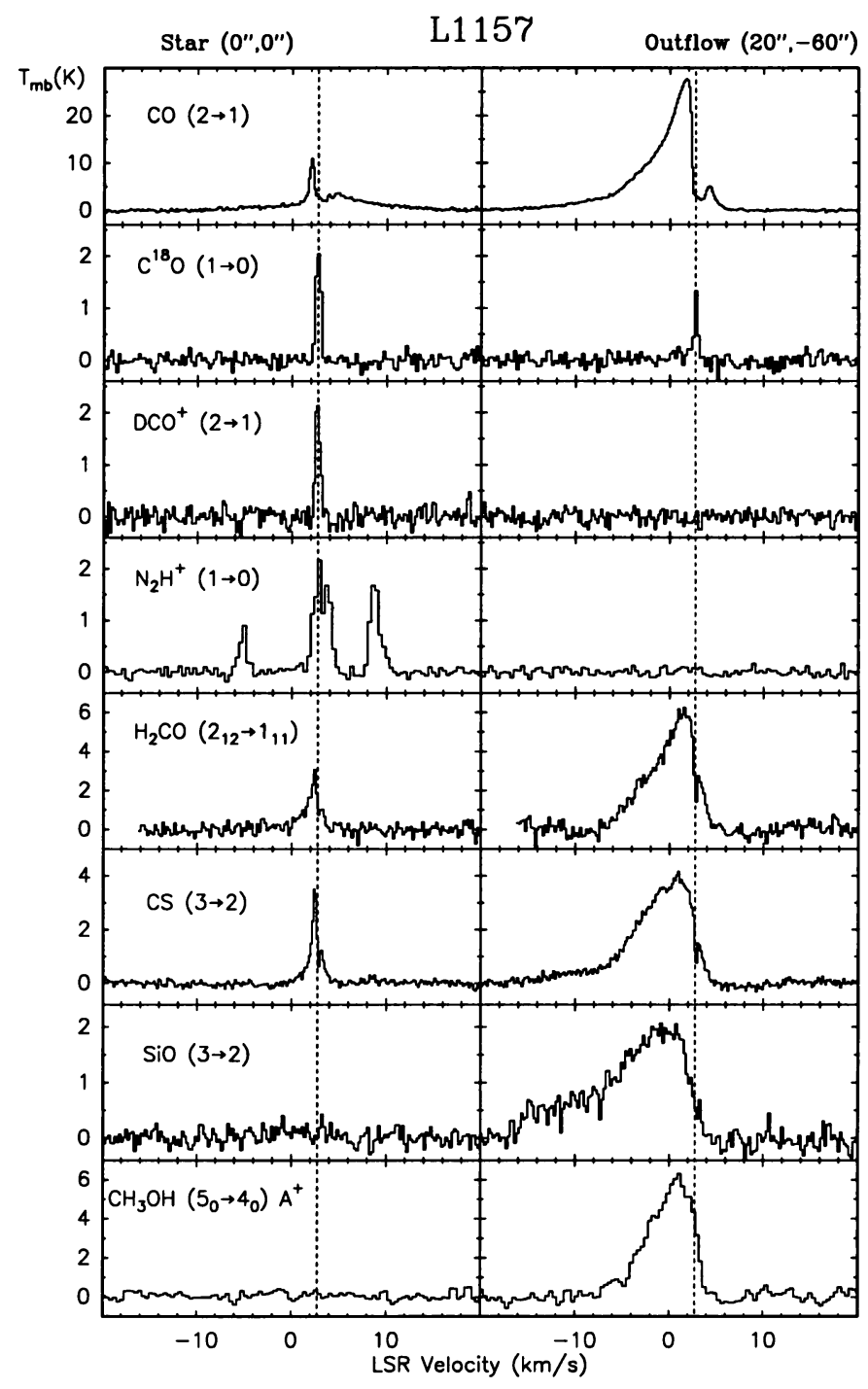

Figure 2. Molecular line profiles observed towards two representative positions in the L 1157 outflow: the $(0,0)$ position on the central star and position $\left(20^{\prime \prime},-60^{\prime \prime}\right)$ on one of the bow shocks in the blueshifted lobe. The lines toward the star positions are narrow, since they arise from quiescent material associated with the dense core. The lines observed toward the outflow are very broad and blueshifted with respect to the ambient gas velocity (marked with a dashed line). 
only observed toward the cold gas condensation around the exciting source, whereas some other molecules (such as $\mathrm{SiO}$ ) only trace the warm gas in the shock. This result is important for studies of the structure of the central infalling condensation, since one can avoid the outflow contribution to the line profiles by observing the source vicinity in lines of $\mathrm{C}_{3} \mathrm{H}_{2}, \mathrm{~N}_{2} \mathrm{H}^{+}, \mathrm{H}^{13} \mathrm{CO}^{+}$, and $\mathrm{DCO}^{+}$. This does not mean that the outflowing gas is fully free of these molecules, but the column densities are insufficient to produce significant wings in the profiles.

All the other detected molecules $\left(\mathrm{SiO}, \mathrm{CH}_{3} \mathrm{OH}, \mathrm{H}_{2} \mathrm{CO}, \mathrm{HCO}^{+}, \mathrm{HCN}\right.$, $\mathrm{HNC}, \mathrm{CN}, \mathrm{SO}, \mathrm{SO}_{2}, \mathrm{CS}, \mathrm{OCS}, \mathrm{H}_{2} \mathrm{~S}, \mathrm{H}_{2} \mathrm{CS}$ ) present emission in the outflow.

\section{Spatial distribution of different molecular species in $\mathrm{L} \mathbf{1 1 5 7}$}

To better characterize those gradients in the chemical composition of the gas, we have recently mapped the most interesting molecular lines with the IRAM 30-m telescope. Some preliminary maps are presented in Fig. 3 . Note the large differences observed in the distribution of the different molecules. In particular the emission of $\mathrm{N}_{2} \mathrm{H}^{+}$is well concentrated around the exciting object, allowing the study of the protostellar vicinity without any contamination from outflow emission.

A first interesting result from the mapping observations concerns the relative intensity of the lines at the two positions B1 and B2, in the blueshifted lobe. Both positions are almost equally strong in the $\mathrm{CO}$ isotopes, $\mathrm{SiO}$ and $\mathrm{CH}_{3} \mathrm{OH}$. However, the peak $\mathrm{B} 1$ is more prominent in $\mathrm{H}_{2} \mathrm{CO}, \mathrm{HCO}^{+}$, and $\mathrm{CN}$, whereas the $\mathrm{B} 2$ peak is somewhat brighter (in peak intensity) in most sulphur-bearing molecules like $\mathrm{SO}$ and $\mathrm{SO}_{2}$. On the other hand, the behavior of CS is different from the other sulphuretted molecules, and seems more similar to $\mathrm{SiO}$ or $\mathrm{H}_{2} \mathrm{CO}$. All these striking differences cannot be explained as a mere result of possible differences in the excitation. Important chemical differences do exist between positions B1 and B2.

The abundances of the different molecules have been estimated from the ratios of the column densities to the $\mathrm{CO}$ column density, and by assuming a standard $\mathrm{CO} / \mathrm{H}_{2}$ ratio. The $\mathrm{CO}$ column densities in the quiescent gas and in the outflow have been derived from the $\mathrm{C}^{18} \mathrm{O}$ lines and the $\mathrm{CO}$ line wings, respectively, by assuming optically thin emission and thermalization. For the other molecules we used a Large-Velocity-Gradient (LVG) code, or we did similar assumptions as in the case of CO. The enhancement factors of the different species in the shock are thus estimated as the ratios of the abundances in the shock to those estimated in the quiescent gas. We next discuss some particular molecules, devoting special attention to the species that are thought to be abundant in the dust grain mantles, since such molecules are expected to be desorbed from grains by the shocks. 
L1157. Blue lobe.

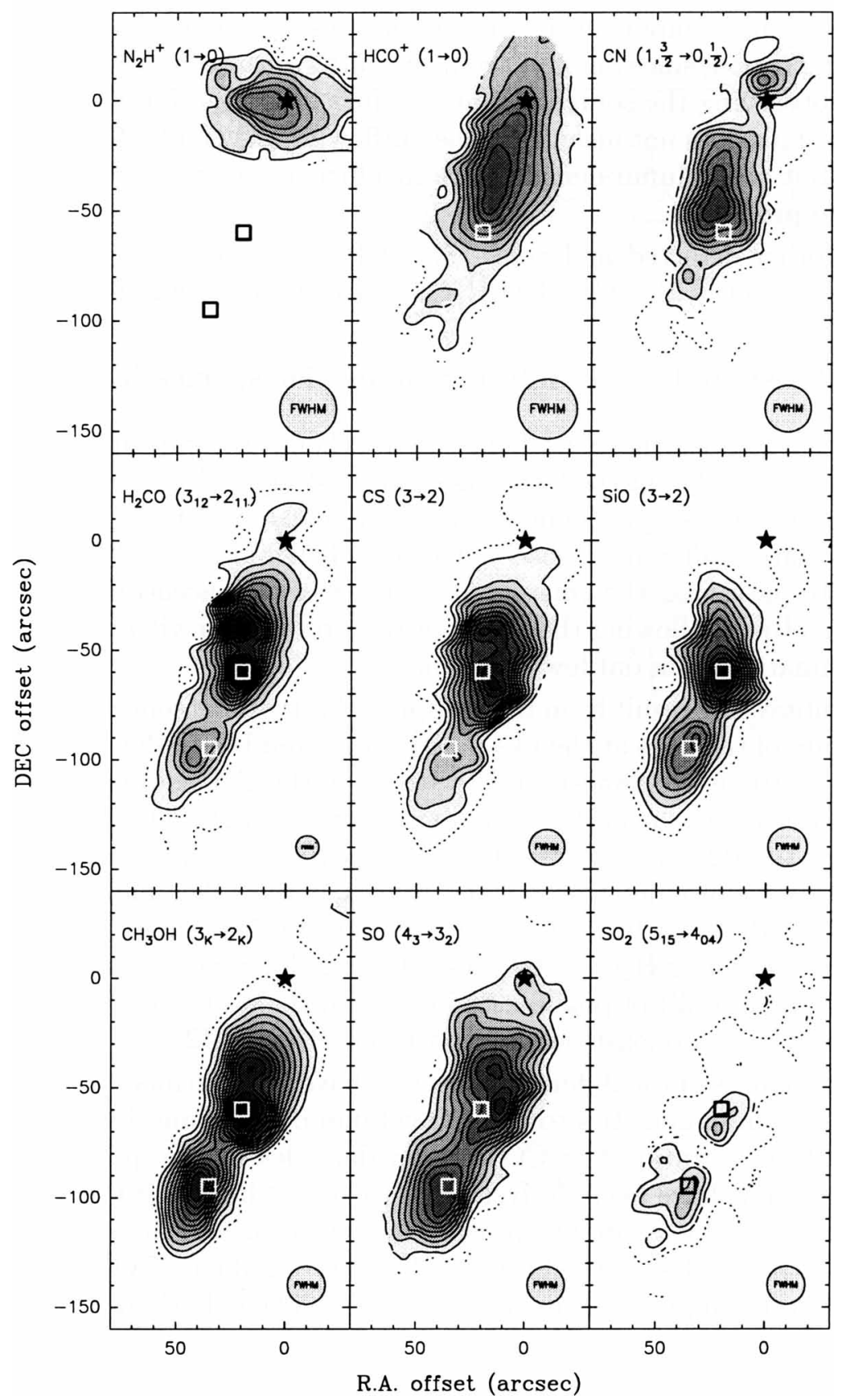

Figure 3. Map of integrated intensities of different molecular lines in the L 1157 outflow obtained at the IRAM 30-m telescope. The white squares mark the positions B1 and B2 where an initial molecular line survey was carried out (see text). 


\section{Chemical processes}

The molecular species detected in solid phase in star forming regions include $\mathrm{H}_{2} \mathrm{O}, \mathrm{CH}_{3} \mathrm{OH}, \mathrm{CO}, \mathrm{CO}_{2}, \mathrm{CH}_{4}$, and OCS. Methanol $\left(\mathrm{CH}_{3} \mathrm{OH}\right)$, with an abundance of a few percent, is thought to be the most abundant molecule in ices, after water (Allamandola et al. 1992). Indeed, the millimeter-wave observations of L 1157 (Bachiller et al. 1995) show that the abundance of methanol is enhanced by a factor of 400 in the shock (see also Avery \& Chiao 1996). Such methanol enhancements have been observed in some other outflows (Sandell et al. 1994; Bachiller et al. 1995; Garay et al. 1996).

There is increasing evidence that high abundances of methanol in gas phase are able to drive a chemistry completely different from the usual chemistry operating in dark clouds. In particular, laboratory experiments show that a significant fraction of the methanol released to gas phase could produce $\mathrm{H}_{2} \mathrm{CO}$ in a relatively short time (e.g. Bernstein et al. 1995). Solid formaldehyde $\left(\mathrm{H}_{2} \mathrm{CO}\right)$ has also been recently discovered in the vicinity of protostars (Schutte et al. 1996). Our observations of L 1157 indicate that $\mathrm{H}_{2} \mathrm{CO}$ in enhanced by a factor close to 100 in the shocked region. The chemistry of formaldehyde is probably closely related to that of methanol in both solid and gas phases.

The chemistry of sulphur is also severely affected by shocks (e.g. Pineau des Forêts et al. 1993), and SO has been found to be enhanced in several molecular outflows (Martín-Pintado et al. 1992; Schmid-Burgk \& Muders 1995; Chernin et al. 1994). The observations of $\mathrm{L} 1157$ reveal significant enhancements of $\mathrm{SO}, \mathrm{SO}_{2}, \mathrm{OCS}, \mathrm{H}_{2} \mathrm{~S}$, and $\mathrm{H}_{2} \mathrm{CS}$ in the shocked region, with the peculiarity, as mentioned above, that the $\mathrm{B} 2$ peak is richer in most sulphur-bearing molecules. It is interesting to note that the $\mathrm{SO} / \mathrm{H}_{2} \mathrm{~S}$ and $\mathrm{SO}_{2} / \mathrm{H}_{2} \mathrm{~S}$ ratios change significantly from position $\mathrm{B} 1$ to $\mathrm{B} 2 . \mathrm{H}_{2} \mathrm{~S}$ is probably formed by grain-surface reactions (Duley et al. 1980) and could be directly released from the mantles. Once released into the gas phase, $\mathrm{H}_{2} \mathrm{~S}$ is expected to produce $\mathrm{SO}$ and $\mathrm{SO}_{2}$ very quickly (in a few $10^{3} \mathrm{yr}$ ) via reactions with $\mathrm{H}, \mathrm{OH}$, and $\mathrm{O}_{2}$ (Millar 1993). Thus, the differences in the $\mathrm{SO}, \mathrm{SO}_{2}$, and $\mathrm{H}_{2} \mathrm{~S}$ abundances between $\mathrm{B} 1$ and $\mathrm{B} 2$ could be related to their different ages. It is indeed expected that $\mathrm{B} 2$ is older than $\mathrm{B} 1$, since B2 is more distant to the central star. Another attempt to use the sulphur chemistry as a rough clock in star forming regions has recently been done by Charnley (1997) to explain observations of the Orion Hot Core.

Molecular spectroscopy is also a powerful tool to investigate the physical conditions in the shocked gas. As discussed above, $\mathrm{NH}_{3}$ provides good estimates of the kinetic temperature. Once the temperature is known, multiline studies of $\mathrm{SiO}$ and $\mathrm{CH}_{3} \mathrm{OH}$ provide reliable estimates of the volume densities. In the case of the L 1157 bow shock, the density is found to be 
in excess of $10^{6} \mathrm{~cm}^{-3}$ (Bachiller et al. 1993, 1995). The region emitting the radio lines is significantly colder $\left(\mathrm{T}_{K} \sim 10^{2} \mathrm{~K}\right)$ than the gas traced by the near infrared lines of $\mathrm{H}_{2}$ (in which $\mathrm{T}_{K} \sim$ a few $10^{3} \mathrm{~K}$ ). However, the images of the $\mathrm{L} 1157$ outflow in the $\mathrm{H}_{2}$ lines near $2.1 \mu \mathrm{m}$ (Davis \& Eisloeffel 1995) also reveal the bow shock structures similar to those seen in $\mathrm{CO}$ and $\mathrm{NH}_{3}$. It seems that sharp gradients in the kinetic temperature are present across the bow shocks.

\section{References}

Allamandola, L.J., Sandford, S.A., Tielens, A.G.G.M., Herbst T. 1992, ApJ 399, 134

Avery, L. W., Chiao, M. 1996, ApJ 463, 642

Bachiller, R. 1996, ARAA 34, 111

Bachiller, R., Liechti, S., Walmsley, C.M., Colomer, F. 1995, A\&A 295, L51

Bachiller, R., Martín-Pintado, J., Fuente, A. 1991, A\&A 243, L21

Bachiller, R., Martín-Pintado, J., Fuente, A. 1993, ApJ 417, L45

Bachiller, R., Pérez Gutiérrez, M. 1997, in preparation

Bernstein, M.P., Sandford, S.A., Allamandola, L.J., Chang, S., Scharberg, M.A. 1995, ApJ 454, 327

Blake, G.A., van Dishoeck, E.F., Jansen, D.J., Groesbeck, T.D., Mundy, L.G. 1994, ApJ 428,680

Blake, G.A., Sandell, G., van Dishoeck E.F., Groesbeck, T.D., Mundy, L.G., Aspin, C. 1995, ApJ 441, 689

Bontemps, S., André, P., Terebey, S., Cabrit, S. 1996, A\&A 311, 858

Charnley S.B. 1997, ApJ, in press

Chernin, L.M., Masson, C.R., Fuller, G. 1994, ApJ 436, 741

Davis, J.J., Eisloeffel, J. 1995, A\&A 300, 851

Duley, W.W., Millar, T.J., Williams, D.A. 1980, MNRAS 192, 945

Dutrey, A., Guilloteau, S., Bachiller, R. 1997, A\&A, in press

Garay, G., Koehnenkamp, I., Rodríguez, L.F. 1996, The ESO Messenger 84, 31

Gueth, F., Guilloteau, S., Bachiller, R. 1996, A\&A 307, 891

Gueth, F., Guilloteau, S., Dutrey, A., Bachiller, R. 1997, A\&A, in press

Martín-Pintado, J., Bachiller, R., Fuente, A. 1992, A\&A 254, 315

McMullin, J.P., Mundy, L.G., Blake, G.A. 1994, ApJ 437, 305

Millar, T.J. 1993, in Dust and Chemistry in Astronomy, eds. T.J. Millar \& D.A. Williams (Kluwer, Dordrecht)

Mikami, H., Tomofumi, U., Yamamoto, S., Saito, S. 1992, ApJ 392, L87

Pineau des Forêts, G., Roueff, E., Schilke, P., Flower, D. 1993, MNRAS 262, 915

Sandell, G., Knee, L.B.G., Aspin, C., Robson, I.E., Russell, A.P.G. 1994, A\&A 285, L1

Schmid-Burgk, J., Muders, D. 1995, in Stellar and Circumstellar Astrophysics, eds. G. Wallerstein, A. Noriega-Crespo (ASP Conf. Ser., San Francisco)

Schutte, W.A., Gerakines, P.A., Geballe, T.R., van Dishoeck, E.F., Greenberg, J.M. 1996, A\&A 309, 633

Tafalla, M., Bachiller, R. 1995, ApJ 443, L37

Tafalla, M., Bachiller, R. 1997, unpublished observations

Umemoto, T., Iwata, T., Fukui, Y., Mikami, H., Yamamoto, S., Kamaya, O., Hirano, N. 1992, ApJ 392, L83

van Dishoeck, E.F., Blake, G.A., Jansen, D.J., Groesbeck, T.D. 1995, ApJ 447, 760

Zhang, Q., Ho, P.T.P., Wright, M.C.H., Wilner, D.J. 1995, ApJ 451, L71 